\title{
Ueber Fumarsäure und optisch-unwirksame Aepfelsäure aus Glycerinsäure ;
}

\section{von $A$. Werigo und Tanalar.}

(Eingelaufen den 9. October 1874.)

Durch vorhergehende Untersuchungen hat einer von uns mit den Herren $0 \mathrm{kulitsch}$ und Werner gezeigt, dafs bei Einwirkung von $\mathrm{PCl}_{5}$ auf Glycerinsäure sich Producte bilden, deren Entstehung auf Grund der existirenden Analogieen zu erwarten war, dals nämlich bei Einwirkung von 3 Mol. $\mathrm{PCl}_{6}$ hauptsächlich Bichlorpropionsäurc entsteht, deren Zersetzungsproduct Chloracrylsäure in allen ilıren Eigenschaften mit der von W ich el ha us als $\beta$-Chlorpropionsäure bezeichneten Säure vollstāndig übereinstimmt. Obgleich es als sicher betrachtet werden konnle, dafs die $\beta$-Chlorpropionsäure von Wichelbaus nichts anderes ist als Chloracrylsäure, so hielten wir es doch nicht für überflüssig, seine Angabe über die Umwandlung von $\beta$-Chlorpropionsäure in gewöhnliche Bernsteinsüure eiser genauen Untersuchung zu unterwerfen. Man könnte nämlich noch einwenden, dafs neben der aus Bichlorpropionsāure entstehenden Chloracrylsäure doch noch eine $\beta$-Chlorpropionsäure in den aus Glycerinsäure mittelst $\mathrm{PCI}_{5}$ erhaltenen Producten existire, die in ihren Eigenschaften siclı so nahe an die Chloracrylsäure anschliefse, dafs sie erst durch die Einführung der Carboxylyruppe unterschieden werden könnte. Un diesein Einwande zu begegnen, haben wir die obengenannte Reaction sowohl auf reinen Bichlorpropionsäureäther, als auch auf die bei $150^{\circ}$ siedenden ätherischen Producte angewandt und die dabei entstehenden Säuren genau studirt. Da wir aber keine Unterschiede im Yerhalten zwischen Bichlorpropionsäureäther und dem bei $150^{\circ}$ siedenden Antheil bemerkt 
haben, so werden wir in dieser Abhandlung nur yon Bichlorprupionsãureäther sprechen.

Wichelhaus hat nichts uber den ron ihm befolgten Gang der Abscheidung der vermeintlichen Bernsteinsäure mitgetheilt; um so genauer werden wir ole von uns angewandten Methoden beschreiben.

Durch Ersetzen der beiden Chloratome im Bichlorpropionsāareäther durch Carboxyl könnte eine Säure von der Zusammensetzung $\mathrm{C}_{3} \mathrm{H}_{6}$ (COHO $\mathrm{CO}_{2} \mathrm{O}_{2}$ entstehen. Zieht man aber die Leichtigkeit, mit witcher ein Vlolecul Salzsänre vom Bichlorpropionsäurcather sich abspaltet, in Beiracht, so ist die Bildung vun Fumarsäure möglich. Fafst man am Ende ins Auge noch den leichten Lebergang von Bichlorpropionsäure und von Chloracrylsüure in eine Milchsäure, so ist auch die Bildung von einer Säure von der Zusaminensetzung der Aepfelsäure wahrscheinlich. In Wirktichkeit ist es uns bis jetzt gelungen, zwei von den drøi erwăhnten Säuren zu finden, nümlict. Fumarsäure und dite optisch-unwirksame Aeplelsäure, von Bersteinsäure fiadet sich unter den Producten der Reaction nicht eine Spur. Wir begnugten uns nicht mit einmaliger Austührung des Experiments, sondern wir haben die Reuction mehrere Male wiederholt und haben dabei bedeutende Mengen von Material gebraucht, da wir für jedes Experinent nicht weniger als 50 Grm. des Aethers benutzt haben.

Wir behondelten den Aether mit 2 Mol. Cyankalium in wasseriger und scliwach alkoholischer Losung. In alkohoiischer Lösung geht die Zersetzung leichter und schneller vor sich, die Producte, welche man durch nachherige Zersetzung mit Kalihydrat bekommt sind dieselhen, wie bei wässeriger Lüsung. Die rothbraane Flüssigkeit wurde mil Kalibydral gekocht, bis die Entwickelung von Ammoniak aufhorte, was 10 bis 15 Stuaden duuerte. Die alkalische Flüssigheil wurde uun mit Schwefelsäure oder Essigsaure neutralisirt, mit kleiven Ueber- 
und optisch-unwirksame Aepfelodiure aus Glycorinsäure. 369

schưfs von Săure versetzt and dann sechs Mal mit Aether ausgezogen. Nach dem Abdestilliren des Aethers aus den ätherischen Auszügen bleibt eine kleine Menge eines braun gefärbten krystallinischen Productes, das in kaltem Wasser sehr schwer losslich ist, leichter aber in heifsem Wasser, Alkchol und Aether sich auflost.

Die Krystalle warden in kochendem Wasser gelöst, die Lösung mit Thierkohle (Blutkohle) entfürbt. Beim Abkühlen der farblosen Lösung schieden sich kleine weifse Krystalle der Säure, welche in allen ihren Eigenschaften vollstanndig mit Furnarsãure übereinstimmt, aus. Wir haben die Fumarsäure aus Aepfelsāure dargestellt und die Vergleichung der Eigenschaften und Reactionen der Säuren beiderlei Abkunft liefs keinen $\mathbf{Z w e i f e l ~ u ̈ b e r ~ i h r e ~ I d e n t i t a t . ~}$

Wir haben ferner die Fumarsăure aus Glycerinsăure in Sibersalz umgewandelt und dieses analysirt. Die Umwandlung geschah in der Art, dafs wir die Säure in heifsem Wasser lōsten, die Lösung genau mit Katilydrat neutralisirten und mit salpetersaurem Silber versetzten. Die Analyse gab folgende Resultate :

0,127 Grm. Subatane gaben 0,1098 AgCl oder $0,0826369 A_{\mathbb{B}}$, was $65,07 \mathrm{pC} . \mathrm{Ag}$ entspricht.

$0,59 \mathrm{Grm}$. Substanz gaben beim Verbrennen mit Kupforoxyd 0,3108 Kohlenstiura und 0,0487 Wasser.

\begin{tabular}{lcc} 
& Barechnet fir \\
\multicolumn{2}{c}{ Silbe asalz der Fumarsture } & Gofundan \\
C & 14,54 & 14,36 \\
H & 0,60 & 0,91 \\
Ag & 66,45 & 65,07
\end{tabular}

Die Menge der sich bildenden Fumarsāure ist sehr gering, doch haben wir so viel davon gewonnen, dafs wir verschiedene Salze dieser Säure darstellen konnten. Wir inüssen bemerken, wenn man hauptsächlich Fumarsäure darstellen will, so mufs man die alkalisohe Flüssigkeit mit Schwefelsäure 
und micht mit Bssigsäure übersätigen, sonst zieht nachher der Aether Fssigsine und auch ein wenig Aepfelsäure aus; die Anweserhait diesar Säuren hindert das Herauskrystallisiren der Fumargaure.

Die nach dem Ausziehen mit Aether gebliebene saure, von Aether durch Kochen befreite Losung wurde mit Bleizuckerlösung so lange versetat, bis bei weiterem Zusatze kein Niederschlag mehr entstand. Der voluminöse amorphe, im Wasser fast unlösliche Niederschlag wird gut ausgewaschen, mit Wasser vermischt und init Schwefelsaure zersetzt, aber so, dafs keine Schwefelsäure in die Lôsung übergeht. Diese Lōsung enthält eine freie organische Säure, ist aber noch stark gefärbt und mufs einige Male mit Blutkohle gekocht werden, bis sie farblos ist. Dann wird die Lösung genau mit Kalihydrat neutralisirt und mittelst essigsaurem Blei das Bleisalz und mit salpetersaurem Silber das Silbersalz der Säuro niedergescblagen. Die freie Säure krystallisirt bcim Abdamplion ihrer Lösung in kleinen Nadeln, ist sehr leicht löslich in Wasser, aber nicht zerfliefslich und hat alle Eigenschaften der optisch-!mwirksamen Aepfelsäure.

Die Analysc des Blei- und Silbersalzes gab folgende Resultate :

Q,4245 Grm. Bloisaly gaben $0,42 \mathrm{PbSO}_{4}$ oder $60,45 \mathrm{pC}$. $\mathrm{Pb}$.

0,3425 Grm. Bleisale gabei 0,505 PbBO, oder 60,88 pC. Pb.

0,6478 Grm. Bleisalz gaben beim Verbreunun mit Kupferoxyd 0,342 Koblensiure cuder 14;40 pC. Kohlenstrff und 0,075 Wasser oder 1,26 pC. Wasserstoff.

0,585 Grm. Bleisalz gaben 0,2985 Kohlenshure oder 13,91 pC. Koblenstoff und 0,0665 Wasser oder $1,29 \mathrm{pC}$. Wasserstoff.

0,405 Grm. Bleianla gaben 0,1926 Koblenglure oder 14,10 pC. Koblonstoff nnd 0,0405 Waseer oder 1,2 pC. Wasserstoff.

Berechniet für

Bluisale der Aopfeletture

C

14,16

B $\quad 1,18$

Pt 61,00

$\overbrace{14,40} \begin{array}{rrr}13,91 & 14,10 \\ 1,26 & 1,29 & 1,20 \\ 1,46 & 60 ; 83 & -\end{array}$


und optisch-unvoirlesame Aepfelsaure aus Glycerineture. 371.

0,4189 Grm. Bilberule gaben 0,841 Cblorsilber oder 61,84 pC. Ag. 0,9004 Grm. Bilbersalz gaben 0,2473 Chlorsilber oder 61,84 pC. Ag. 0,4087 Grm. Silbersalz gaben beim Verbrenned mit Kupferoxyd 0,2103 Kohleneluri oder 14,0 pC. Koblenstoff und 0,0485 Waster ofer 1,18 pC. Wasserstoff.

\begin{tabular}{|c|c|c|c|}
\hline \multicolumn{2}{|c|}{$\begin{array}{l}\text { Berechnet fur } \\
\text { Gilberulz der Aopfelesure }\end{array}$} & \multicolumn{2}{|c|}{ Gefanden } \\
\hline $\mathbf{C}$ & 13,78 & 14,00 & - \\
\hline H & 1,14 & 1,18 & - \\
\hline$\Delta_{\mathbf{B}}$ & 62,07 & 61,94 & 61,8 \\
\hline
\end{tabular}

Die Analyse zoigt also, defs die Säure die Zusammensetzung der Aepfelsäure hat, um aber die vollständige Ueberzeugung, dafs sie einr optisch-unwirksame Aepfelsäure ist, zu gewinnen, haben wir diese letzte Săure aus gewöhnlicher Aepfelsäure nuch der Methode von Pas teur dargestellt und die Eigenschaften einer Reihe von Salzen aus den Säuren beiderlei Abkunft genau verglichen, dabei consiatirten wir die vollkommene Identität beider Săuren. Da die Salze von optischunwirksamer Aepfelsaure sehr wenig bekannt sind, so wollen wir hier kurz erwähnen, dafs die leicht löslichen Alkallsalze schwer krystallisiren, sie werden sämmtlich aus wässeriger Lösung durch Alkohol als flockige amorphe Niederschläge gefällt; ebenso verhalten sich Kupfer- und Nickelsalze. Die wässerige Lōsung des Zinksalzes trübt sich beim Erhitzen, scheidet einon amorphen Niederschlag aus, der beim Abkühlen witder verschwindet. Blei-, Silber- und Quecksilbersatze sind fast unloslich in Wasser, dic beiden letzten werden dadurch leicht zersetzt.

Funarsäure und optisch-unwirksame Aepfelsãure sind also die Producte der oben beschricbenene Reaction, sie deriviren durch einfache Gleichungen von Bichlorpropionsăure und Chloracrylsäure. Von Bernsteinsâure ist keine Spur vorhanden, das was Wichelhaus dafür gehalten bat ist mōglicherweise ein Genvisch von Fumarsäure und Aepfelsäare; die von 
ibm a.ıgegebenen Eigensohaften der Säure lasseu sich gut auf Fumırsäure beziehen.

Da diese Arbeiten in allen den Richturigen, welche in dieser und den vorhergehenden Abhandlungen angebahnt sind, fortgesetzt werden, so begnügen wir uns einstweilen, zu den schon gezogenen Schlüssen noch den vollständig berechtigten hinzuzufügen, dafs die $\beta$-Chlorpropionsäure und der $\beta$-Chlorpropionsäureâther von $W$ ic hel ha us nicht existiren.

Odessa, Universitātslaboratorium, den 5. October 1874.

\section{Ueber die Darstellung von Aethylchlorür und seinen Homologen; von Charles E. Grooes *).}

Zur Darstellung von Aethylchlorür werden hauptsächlich 2wei Methoden angewendet :

1) Alkohol wird mit trockenem salzsaurem Gas gesältigt, einige Zeit stehen gelassen und dann aus dem Wasserbad destillirt;

2) Destillation einer Mischung von Alkohol, Schwefelsäure und Kochsalz.

Für die Bereitung gröfserer Mengen sind beide Verfahren wenig brauchbar; bei dem ersteren wird nur ein verhältnilsmäfsig kleiner Theil des Alkohols, etwa $15 \mathrm{pC}$., in Chlorür verwandelt, wälırend Ströme von Salzsãure entweichen; das zweite hat neben dieser geringen Ausbeute noch den weiteren

-) Uebersetzing eines rom Verfasser eingosendeten Separatabdrucke aus Chem. Soc. Joun., Juti 1874. 